\title{
1987 Conference on Dynamic Patterns in Complex Systems
}

\author{
JANET METCALFE and JOHN MERRILL \\ Indiana University, Bloomington, Indiana
}

\begin{abstract}
Conference on Dynamic Patterns in Complex Systems, Fort Lauderdale, Florida, October 21-23, 1987.
\end{abstract}

INVITED SPEAKERS: Avis Cohen, Section of Neurobiology and Behavior, Cornell University; Doyne Farmer, Center for Nonlinear Studies and Theoretical Division, Los Alamos National Laboratory; Ivan Giaever, General Electric Corporate Research \& Development Center; Ary Goldberger, Beth Israel Hospital, Harvard Medical School; Stephen Grossberg, Center for Adaptive Systems, Boston University; Nancy Kopell, Department of Mathematics, Boston University; Rolf Landauer, IBM Thomas J. Watson Research Center; Benoit B. Mandelbrot, IBM Research Center and Harvard University; George J. Mpitsos, Mark O. Hatfield Marine Science Center, Oregon State University; Norman H. Packard, Center for Complex Systems Research, University of Illinois at Urbana-Champaign; Itamar Procaccia, Chemical Physics Department, The Weizmann Institute of Science; Paul E. Rapp, Department of Physiology, The Medical College of Pennsylvania; Robert Rosen, Department of Physiology \& Biophysics, Dalhousie University; Otto Rössler, Institute for Physical \& Theoretical Chemistry, University of Tubingen; Gregor Schöner, Center for Complex Systems, Florida Atlantic University; Allen I. Selverston, Department of Biology, University of California-San Diego; Hiroshi Shimizu, Department of Pharmaceutical Sciences, University of Tokyo; Harry L. Swinney, Center for Nonlinear Dynamics and Department of Physics, The University of Texas at Austin; Harold Szu, Naval Research Laboratory; Esther Thelen, Department of Psychology, Indiana University; Michael Turvey, Center for the Ecological Study of Perception and Action, University of Connecticut and Haskins Laboratories; William Warren, Departments of Psychology and Cognitive Science, Brown University; Bruce J. West, Division of Applied Nonlinear Problems, University of California-San Diego. ORGANIZERS: J. A. S. Kelso, Center for Complex Systems, Florida Atlantic University; A. J. Mandell, Laboratory of Biological Dynamics and Theoretical Medicine, University of California, San Diego.

This conference was dedicated to Hermann Haken on his 60 th birthday. Haken did fundamental theoretical work on the laser, and later expanded this work into a field of mathematico-philosophical inquiry called synergetics, of which the study of complex dynamical systems is a part.

J. Metcalfe is in the Department of Psychology, and J. Merrill is in the Department of Computer Science, at Indiana University, Bloomington, IN 47405.
A quotation from Haken conveys some of the ambition, flavor, and scope of the conference:

The spontaneous formation of well-organized structures out of germs or even out of chaos is one of the most fascinating phenomena and challenging problems scientists are confronted with. Such phenomena are an experience of our daily life when we observe the growth of plants and animals. Thinking of much larger time scales, scientists are led into the problems of evolution, and ultimately, of the origin of living matter.... It has become more and more evident that there exist numerous examples in physical and chemical systems where well-organized spatial, temporal, or spatiotemporal structures arise out of chaotic states. Furthermore, as in living organisms, the functioning of these systems can be maintained only by a fluctuation of energy (and matter) through them. In contrast to man-made machines, which are devised to exhibit special structures and functionings, these structures develop spontaneously-they are self-organizing. It came as a surprise to many scientists that numerous such systems show striking similarities when passing from the disordered to the ordered state. This strongly indicates that the functioning of such systems obeys the same basic principles. (Haken, 1983, p. viii)

In his opening remarks, Kelso proposed an agenda for the conference-to begin to delineate a common vocabulary for describing and understanding complex dynamical processes across the many fields of research in which these common concepts are being studied. Such a vocabulary did emerge, shared by a diverse community of researchers: biologists and psychologists observing movement in animals from invertebrates to human babies, physicists studying single mammalian cells, biomedical scientists looking at the dynamics of sudden heart failure, and mathematicians devising abstract models. These scientists all found that the language of nonlinear dynamics was well suited to expressing their observations and hypotheses.

Complex dynamics is the study of deterministic systems evolving over time, in which the variables are coupled to one another in a nonlinear fashion. One natural area of application is the behavior of abstract neural networks, whose outputs form just such a system, since they are dependent upon one another, but not in a simple, linear way. Usually, small changes in the defining (or, formally, "control") parameters of a complex dynamical system 
yield only gradual changes in behavior. But, in contrast to the situation in linear systems, values of the control parameters exist at which the behavior changes suddenly. These changes-"bifurcations" and "phase transitions"- generated much interest at the conference, since they offer a model for spontaneous self-organization, a phenomenon of great scientific concern. Bifurcation occurs when one stable configuration divides into two new configurations. Phase transitions occur when an organization of the system first becomes unstable and then restabilizes in a new mode. Another characteristic property of complex dynamical systems that received considerable attention was "chaos" and the related study of "fractals." Chaotic behavior is characterized by extreme complexity and apparent impenetrability. A particularly amusing discussion of chaos (which will be summarized below) was given by Rössler, who talked about a familiar chaotic system: puff pastry.

The first day of the conference was dominated by discussion of the emergence of behavior in networks of neurons, a particular complex nonlinear system of focal interest to many of the participants. Mpitsos and Selverston discussed the structure of central pattern generators in invertebrates. There was no trace of a homunculus in their models; instead, these models looked like circuit diagrams whose behavior could be qualitatively altered by the presence or absence of different chemiconeuromodulators. Cohen gave a talk on observations of periodic behavior in the isolated lamprey spinal cord; Koppel followed immediately with a discussion of mathematical models of such behavior. Grossberg, Shimizu, and Szu gave informative and interesting presentations on their work on abstract neural networks consisting of systems of nonlinear, coupled differential equations.

Szu incited a lively discussion with the question "Just what is a neural network?' While this may seem a trivial question in the strictly physiological sense, as Cohen and Mpitsos pointed out, it is not at all trivial in a cognitive or computational context. What are the essential features of the human brain that allow separation into different functional systems, or modules? What are the implications, in the cognitive realm, of the precise details of the interactions among neurons? Szu noted that there are (at least) three distinctly different levels of representation, which have been used interchangeably in the literature, all called neurons. He suggested that we refer to these representations as (1) neurons, (2) neurons, and (3) neurons.

Szu equated the microscopic Type 1 neurons with hippocampal neurons. Presumably, in order for patterns and representations to emerge from this level, many such units must act in concert. Type 1 neurons yield readily to a synergetic analysis. The second type, the neuron, is akin to a sensorimotor neuron that already encodes compressed information about macrounits. Finally, the third typethe neuron, the representation used in propositional models-he equated with the grandmother cell, complete with internal memory and complex decision processes. Szu argued that the indiscriminate use of terminology with such radically different denotations causes enormous communication problems in the cross-disciplinary study of neural networks. He also proposed a generic definition of a neural network as (1) nonlinear, (2) nonlocal, (3) nonstationary, and (4) nonconvex. As one participant quipped, this nondefinition leaves considerable room for expansion.

Complex dynamical systems are being applied in the fields of developmental psychology and kinematics. Thelen elaborated on some of the implications of nonlinear dynamical systems in infant motor development. She has discovered how small changes in the environment can radically alter the organization of coordinated walking at critical times, and she posits that this reflects the instability of a dynamical system near a phase transition. In studying the gait changes in adult humans going from bipedal climbing to quadrupedal scrambling, Warren used a dynamical analysis to reveal dimensionless invariants relating the person's body size to the slope of the incline. This analysis generated predictions about the variability of the movements near the changing points and about the asymmetry of the change (hysteresis)-novel predictions that were borne out by the data. Turvey argued that an adequate model of behavior must include and reflect the interaction between the animal and its environment. He took as one of his examples the problem of a gannet's movement as it approaches the surface of a lake. Until it actually commits to either landing on or diving through the surface, there is no way to predict the movement. The bird instantiates its intention by considering its entire flow field. Turvey provided a dynamical model for the means by which this occurred.

Schöner and Kelso reported on work investigating phase transitions in the control of hand movements. Kelso's group has studied a paradigm in which people are required to rhythmically move both hands in synchrony, maintaining a fixed relationship between the two. As a subject increases the speed of the movement, a point is reached at which the organization quickly and dramatically switches from "American" to "British" windshield wiper mode (i.e., from out-of-phase to in-phase). This group has constructed an elegant model that explains most of the data within this paradigm. Schöner and Kelso reported on the impact of classical cognitive variables-intention and memory-within what has heretofore been considered a purely automatic system. The incorporation of intentionality within this tightly controlled and well-understood system shows great promise, and also suggests a bridge between dynamical systems theory and cognitive science, where constructs such as goals and intentions are commonplace. The instantiation of psychological parameters may be of enormous importance in enhancing our understanding of complex, learned coordinations, such as pi- 
ano or cello playing, that develop out of more primitive, innate, phasic organizations. The development of complex learned coordinations is, of course, a classic unsolved problem.

There were many talks of general interest. Landauer gave a talk on the importance of the "blowtorch" theorem: In order to understand how a system will behave, one needs to have information not only about the attractiveness of some stable states of the system, but also about the noisiness of the terrain between the current state and any other stable state. This is called the blowtorch theorem because the variance of this noise might be increased by external factors (like external heating by a blowtorch). Farmer presented a technique whereby one can make local predictions within a nonlinear system that surpass predictions made with standard (linear) techniques. He noted that this nonlinear analysis did not improve stock market predictions, even locally, alas. Mandelbrot, most famous for the discovery of fractals, noted that the recent erratic behavior of the stock market is essentially random, and perhaps characterized by a Lévy random walk. He also provided some reminiscences about the early history of fractals, the study of which figured large at the conference. Fractals were entertainingly explained in a talk by Rössler concerned with chaos in French puff pastry. Puff pastry is made by taking dough and spreading a layer of butter on top. This is then folded over, to yield a four-layer sandwich, which is then folded over and rolled out to yield an eight-layer sandwich, which is then folded over, and so on. After about 25 iterations of this procedure, the dough consists of layers whose thickness is less than that of a single atom-the system is thoroughly mixed. Now consider the path of any single molecule of the initial sandwich. It is completely determined by the initial conditions-yet molecules that are arbitrarily close together at one time can wind up on opposite sides of the sandwich at a later time. This kind of unpredictable, yet deterministic, mixing is the hallmark of chaos. Fractals can be derived from such systems by many means. For instance, if the position of any molecule of butter were plotted against its own position one fold later, the curve generated over the set of all folds would be a fractal. West presented an elegant analysis of the fractal structure of the human lung. From strikingly simple initial assumptions, he derived a model of the branching number of the bronchial passages that was in accord with the observed frequencies. Noting also that the proposed fractal structure of the lungs predicts an observed tolerance to developmental error (which is not accounted for by other models), he argued compellingly for the evolutionary significance of such a model.

The concepts from this conference have practical implications. Rapp detailed some of the clinical aspects of dynamical analyses. For example, EEG differences between vigilant and relaxed states were apparent with fractal analysis but not with more traditional analyses. He sug- gested that the sensitivity of this measurement technique, as well as the fact that it provides results quickly and with less data than other analyses require, positively indicates its potential as a tool for anticipating pilot blackout, predicting the onset of epileptic seizures, or monitoring anesthesia level. Goldberger applied a fractal model to cardiac arrhythmia, reaching the surprising conclusion that death is preceded by increased, not decreased, periodicity. Mandell applied a fractal analysis to the tertiary structure of proteins, which is one of the most difficult problems in protein chemistry today. Using the nonlinear dynamical techniques, he obtained amazing, and easily computable, invariants of protein structure that allowed him to actually synthesize biologically active, but completely artificial, proteins. This work could have major implications.

The presentations given at the Conference on Dynamic Patterns in Complex Systems indicate that a Kuhnian paradigmatic shift is taking place in medicine, physics, chemistry, biology, and mathematics. The implications of this work within the field of psychology are not yet apparent. Although there are excellent introductions to the general topic of the behavior of complex dynamic systems (particularly Abraham \& Shaw, 1985), it frequently seems that workers in the field of complex dynamical systems delight in speaking a language that is jargon-ridden and incomprehensible to the uninitiated. It is not clear whether such concepts as "bifurcation," "phase transition," "chaos," "fractals," and "entrainment," have general application within psychology, although their usefulness is beginning to be seen in the study of motor movements. It is also unclear how, or indeed whether, the constructs from nonlinear dynamics map onto traditional psychological issues, or what new insights such a mapping might yield. What is clear is that the translation will be exceptionally difficult, and perhaps risky, for the psychological theorist.

But consider the possible rewards. First, nonlinear models, which are more powerful than linear models, allow explanation of types of behavior and cognitive processes that have resisted interpretation within linear frameworks. For instance, it is impossible to explain the spontaneous emergence of structure in a linear model, although this kind of sudden self-organization is almost certainly widespread in human thinking and behavior. Investigation of the possibility of such phenomena within psychology has been restricted, perhaps because of a false belief that emergence of new structure was not only unscientific but also theoretically impossible. Second, the fact that dynamical systems are intrinsically temporal corresponds to the inherently temporal nature of human cognition. Thus, in building models in this framework, we need not add the temporal dimension in an ad hoc manner, as is usually done with linear models. Third, the ability to deal with complex systems in a natural and analytical way provides a means whereby the brain-in all its 
manifest complexity - can be modeled in a principled manner. Fourth, and finally, new predictions about the variables that may be critical to a system, such as the amount, kind, and timing of noise, are already being investigated in other domains, and may prove important in psychological understanding as well.

There are those who would argue that the human being is the epitome of complex patterning dynamical systems. If so, then the potential enhancement of our understanding of human cognition that will result from the study of synergetics will almost certainly repay the most diligent effort.

\section{REFERENCES}

Abraham, F., \& Shaw, R. (1985). Dynamics: The geometry of behavior (Vols. 1-3). Santa Cruz, CA: Ariel Press.

HAKEN, H. (1983). Synergetics: An introduction. Berlin: SpringerVerlag.

(Manuscript received November 17, 1987.) 